\title{
Carcinoma Cuniculatum of the Foot: A Case Report
}

M. Aboudourib ${ }^{*}$, A. Aarab ${ }^{2}$, M. Gouatri ${ }^{3}$, N. Mansouri ${ }^{2}$, Md El Amrani ${ }^{3}$, O. Hocar ${ }^{1}$, Y. Benchemkha ${ }^{3}$, H. Rais $^{2}$, S. $\mathrm{Amal}^{1}$

${ }^{1}$ Department of Dermatology, University Hospital Mohamed the VI th, School of medecine, Cady Ayyad University, Marrakesh, Morroco

${ }^{2}$ Department of Anatomopathology, University Hospital Mohamed the VI th, School of medecine, Cady Ayyad University, Marrakesh, Morroco

${ }^{3}$ Department of Plastic Surgery, University Hospital Mohamed the VI th, School of medecine, Cady Ayyad University, Marrakesh, Morroco

DOI: $\underline{10.36347 / \text { sjmcr.2020.v08i02.020 }}$

| Received: 06.02.2020 | Accepted: 14.02.2020 | Published: 16.02.2020

*Corresponding author: Maryem Aboudourib

Abstract

Case Report

Verrucous carcinoma is a rare variant of squamous cell carcinoma. It is well differentiated and rarely metastases but can sometimes be very aggressive locally in depth. The paper presents a case report of a 40-year-old man with an enlarging lesion on the heel of his right foot. The patient underwent tumor excision and the histopathological diagnosis was verrucous carcinoma.

Keyswords: Carcinoma_cuniculatum_foot.

Copyright @ 2020: This is an open-access article distributed under the terms of the Creative Commons Attribution license which permits unrestricted use, distribution, and reproduction in any medium for non-commercial use (NonCommercial, or CC-BY-NC) provided the original author and source are credited.

\section{INTRODUCTION}

Le carcinome cuniculatum est un carcinome épidermoïde bien différencié, de bas grade de malignité, qui siège électivement sur le membre inférieur et essentiellement sur la plante du pied (89\%) [1], son pronostic est lié essentiellement à l'extension locorégionale [2].

Nous présentons le cas d'un carcinome cuniculatum du talon traité par exérèse chirurgicale complète.

\section{Cas Clinique}

Il s'agit d'un homme de 40 ans, sans antécédents pathologiques notables, qui consultait pour une lésion verruqueuse du talon droit, évoluant depuis 10 ans dans un contexte de conservation de l'état général. L'examen clinique objectivait une lésion tumorale cutanée arrondie bien limitée surmontée de verrucosités pierreuses, mesurant $5 \mathrm{~cm}$ de grand diamètre, en regard du tendon d'Achille droit, avec une lymphangite (Figure-1), sans adénopathies inguinales. Le reste de l'examen somatique n'objective pas d'anomalie. Une exérèse complète de la lésion était réalisée. L'étude anatomopathologie de la pièce d'exérèse était en faveur d'un carcinome verruqueux (Figure-2). Le bilan d'extension locorégionale et à distance était négatif.

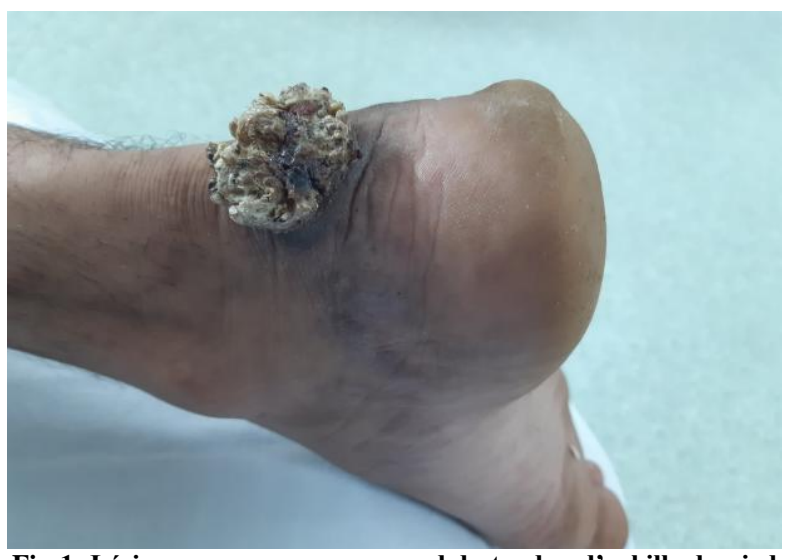

Fig-1: Lésion verruqueuse en regard du tendon d'achille du pied droit

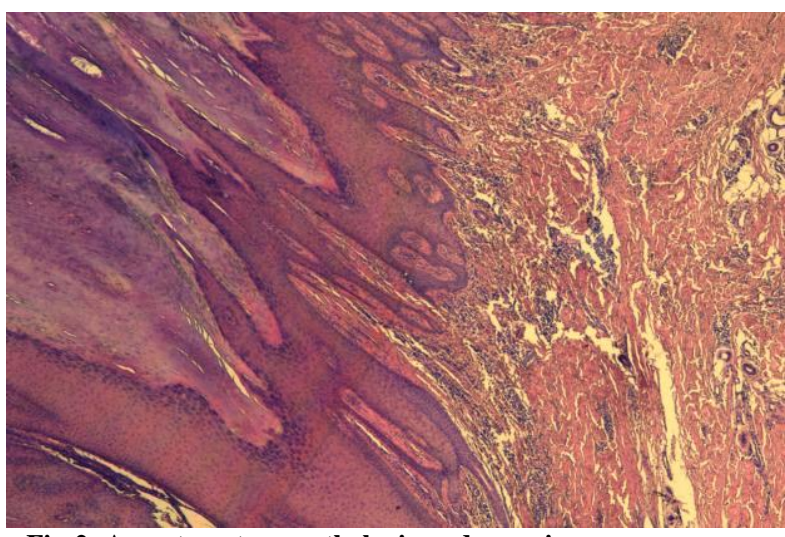

Fig-2: Aspect anatomopathologique du carcinome verruqueux avec extension exophytique et endophytique refoulant le derme 


\section{Discussion}

Le carcinome verruqueux cutané est une tumeur rare, très bien différenciée, à malignité essentiellement locale. Sa pathogénie reste méconnue $[1,3]$. Il est l'apanage du sujet âgé, de sexe masculin [3]. Sur le plan clinique, Il forme une lésion exophytique, verruqueuse qui peut rester longtemps superficielle comme dans le cas de notre patient. La localisation la plus fréquente est la plante du pied. L'atteinte du talon est rapportée dans $16 \%$ des cas dans la littérature [2].

Les traumatismes à répétition, l'irritation chronique, et l'infection par le papillomavirus humain sont les principaux facteurs de risques [4]. Le principal diagnostic différentiel est la verrue plantaire. Sur le plan histologique, le carcinome verruqueux présente deux composantes: endophytique et Exophytique [4, 5]. Le traitement du carcinome verruqueux est chirurgical repose sur l'exérèse complète. Le pronostic est excellent. Les Récurrences sont souvent dues à une exérèse incomplète.

\section{CONCLUSION}

Les carcinomes verruqueux représentent une forme histologique rare bien différenciée du carcinome épidermoïde qui se distingue par ses modalités diagnostiques, thérapeutiques et évolutives particulières. Son traitement est exclusivement chirurgical. Il se base sur une excision large sans curage ganglionnaire. Le pronostic de ces lésions est bon mais grevé par les récidives locales.

\section{RÉFÉRENCES}

1. Yoshitatsu S, Takagi T, Ohata C, Kozuka T. Plantar verrucous carcinoma: report of a case treated with Boyd amputation followed by reconstruction with a free forearm flap. Journal Dermatol. 2002;29:541-542.

2. Kao GF, Graham JH, Helwig EB. Carcinoma cuniculatum (verrucous carcinoma of the skin): a clinico-pathological study of 46 cases with ultrastructural observations. Cancer. 1982; 49:395403.

3. Smith PJ Jr, Hylinski JH, Axe S. Verrucous carcinoma: epithelioma cuniculatum plantar. Journal Foot Surg. 1992;31:324-328.

4. Cassarino DS, DeRienzo DP, Barr RJ. Cutaneous squamous cell carcinoma: a comprehensive clinicopathological classification. Journal Cutan Pathol. 2006;33:191-206.

5. Bhushan M, Ferguson JE, Hutchinson CE, Muston HL. Carcinoma cuniculatum of the foot assessed by magnetic resonance scanning. Clin Exp Dermatol. 2001; 26:419-422. 